\title{
CHOICE OF SENSOR FUSION FRAMEWORK FOR TRAIN POSITIONING SYSTEM
}

\author{
MONISH SENGUPTA \\ Civil, Environmental and Geomatic Engineering, University College London, United Kingdom
}

\begin{abstract}
The general trend in railway length is in overall decline across the country while the passenger demand is growing. To meet this growing demand as well as the capacity to deliver a safe and secure railway, there is a need for use of novel technology to cope with it. Hence the rise of ERTMS and CBTC systems where there is a shift from the conventional fixed block signalling system to moving block signalling system. Sensor fusion technique and sensors sit in the heart of these new systems. Literature review has been carried out to understand the fundamentals of these various sensors currently used on railways. With a combination of literature review and simulation, a study has been carried out via modelling and analysis to explore a better sensor fusion architecture applicable to railway environment which can adapt itself to the various sensor drawbacks. This report summarises the research undertaken and its significant findings in the filter performance with varying measurement and state noise, highlighting the potential for introducing alternative approaches to train positioning that would enable removal of all trackside location correction balises, leading to huge reduction in maintenance cost and more flexibility in future track design.

Keywords: ERTMS, CBTC, sensor fusion, signalling, train control, rail sensors.
\end{abstract}

\section{INTRODUCTION}

Traditional train navigation and control systems use fixed train detection systems such as track circuits and axle counters. These fixed train detection systems form the vital element of the traditional fixed block signalling system.

The capacity of railway is constrained by the fixed train detection system. The fixed train detection system formed by either track circuit or axle counter creates a fixed block. Only one train is allowed within this block which in turn affects the number of trains that can be driven within a given piece of railway. The new digital rail ERTMS level 1 and level 2 or CBTC although uses continuous supervision and automatic train protection, they are still constrained by the use of fixed block signalling system. ERTMS level 3 although uses moving block signalling system, it is constrained by the use of balises on track which restricts the train to fixed infrastructure along the track.

Existing railway on-board sensors used for ERTMS and CBTC and the data fusion technology do not restrict the location uncertainty within the location state of the train. Occasional missing of balises, wheel slip and slide and sensor misalignment leads to unbounded growth in location uncertainty.

As a result, the capacity and hence performance can get affected. Therefore, the railway signalling technology needs to come up with a framework of sensor fusion technology and control mechanism which can be used to reduce the state uncertainty and hence provide an optimal location estimate.

\section{LITERATURE REVIEW}

The sensors currently used for train navigation are tachometers, doppler radar and balise. 


\subsection{Tachometers}

Tachometers are wheel angular speed sensors often used as a primary form of speed measurement device. A whole range of different types of analogue and digital sensors such as capacitative, active and passive electromagnetic tachometers, have been used for some time in rail vehicle applications [1]. They are frequently used due to their robustness and reliability [2].

Discussing about the limitations of a tachometer, Mirabadi et al. [1] suggested parameters limiting the tachometer output accuracy include resolution of the tachometer, sampling frequency, electrical noise, mechanical imperfections and wheel slip and slide.

Wheel slip and slide occurs when the tractive effort exceeds adhesive weight or braking effort exceeds adhesive weight respectively. The adhesive weight is the result of wheel rail friction force. Therefore, during heavy traction, the wheel can rotate freely in an event of wheel slip giving rise to excessive rotational speed. On the contrary, wheel can get locked in the event of wheel slide during heavy braking leading to zero wheel rotation. Geistler and Bohringer [3] while discussing about robust rail speed measurement mentioned that the use of wheel tachometers may lead to degraded operation due to the presence of wheel slip and slide. On the topic of wheel slip and slide, STARS (Satellite Technology for Advanced Railway Signalling) project [4] mentioned that tachometers are the most unreliable of all railway sensors, especially if mounted on a powered axle. The Transit Cooperative Research Programme (TCRP) report [5] on the other hand suggested that errors caused by wheel slip and slide can be minimized by the use of current sophisticated slip-slide control or, where feasible, placing the tachometer on an unmotored axle. Fitting the tachometer on to the unmotored axle however can only restrict wheel slip, but not slide as the braking effort can still lead to measurement inaccuracies.

\subsection{Doppler Radar}

Speed of the train can be measured directly by applying the Doppler principle [1]. The frequency shift as mentioned by Mirabadi et al. [1] is related to the speed by:

$$
F_{d}=(2 V \operatorname{Cos} \gamma) / \lambda
$$

where

$F_{d}$ is the Doppler frequency shift

$V$ is the train speed relative to the ground

$\gamma$ is the radiation angle

$\lambda$ is the transmitted carrier wavelength.

Doppler radar is a non-contact sensor with two microwave antennas which provides good accuracy and reliable output independent of the reflecting surface and the vibration [4]. Mirabadi et al. [6] while discussing about fault tolerant train navigation system identified the Doppler radar as the preferred sensor due to its low amplitude of white noise. Compared to tachometer, they identified Doppler radar to provide higher accuracy data. Heide et al. [7] suggested that use of $24 \mathrm{GHz}$ coded Doppler radar can provide high precision data (within $20 \mathrm{db}$ ) for vehicle position and speed measurement.

Malvezzi et al. [8] on the other hand while discussing odometric estimation for train protection system stated that Doppler radar output is often affected by noise and systematic errors. This has been echoed by Mirabadi et al. [1] who identified the sources of errors due 
to very smooth reflective surface, change in radiation angle due to acceleration and braking action, vibration and radar noise and bias error.

While discussing about the other areas where Doppler effect has been used for speed sensing, Wrobel [9] stated in his research about LIght Detection And Ranging (LIDAR) sensor which also uses Doppler principle to measure the train speed. The doppler shift received by this sensor he suggested can accurately determine the speed by applying eqn (1). Doppler radar is also used in aircraft speed measurement [10].

\subsection{Accelerometers}

Accelerometers are sensors which can measure acceleration. They are not a common sensor that are used on existing speed measurement sensor framework for railways. Some CBTC system uses accelerometer as speed sensing device, but ERTMS in Europe do not prefer the use of accelerometers onboard.

It is a device that can measure acceleration generated by the movement of an object along an axis [11]. Linear accelerometers can be used to measure the acceleration of the vehicle in different dimensions [1].

An important element of this sensor as highlighted by Vettori et al. [12] is that if the sensor axis is not perfectly perpendicular to the gravity vector, the output signal is not null, even if the accelerometer is perfectly still. Mirabadi et al. [6] identified this error as bias error apart from other probable accelerometer sensor errors such as hysteresis error, dead zone, axis alignment error and pivot alignment error.

An important relation between the bandwidth and sensor accuracy has also been identified by Malvezzi et al. [11]. Faster we acquire the accelerometer output, worse the accuracy we get. This is explained by the fact that high sensing frequency requires a high ratio of stiffness to mass and therefore comes at the cost of having poorer sensitivity and resolution [13]. In this context, Endevco [14] in their study on the steps leading to identifying the right accelerometer stated that the frequency response is an important parameter when considering any accelerometer.

The Integrated Electronic Piezoelectric (IEPE) is the most commonly used accelerometer that does not need any external charge amplifier and uses ordinary low-cost cable [14].

\subsection{Balise}

Balise is a transponder fixed on the track by ERTMS and CBTC system to correct the position uncertainty which builds up within the train location subsystem over the time. It provides a position reference and direction information to the train. Balises are magnetically coupled transponders and do not require a constant energy source on their own [15], hence can be considered as passive. However, for ERTMS Level 1 system, balises can be active where they are connected to the signal via a Lineside Electronic Unit. Here the balises are continuously powered.

As pointed out by Mendizabal et al. [16], the balises are placed at approximately regular intervals. The distance between the balises are determined by the speed and complexity of the railway. As it is important for the train to stop precisely at stations, point zones and buffer stops, there can be up to 8 balises required at a minimum interval defined by the design [17]. The main reason of having more than one balise in critical stopping locations is the possibility of train missing to read a balise. Geistler and Bohringer [3] hence suggested a sensor fusion for position estimate in order to reduce the reliance on wayside balise. 


\subsection{Other sensors}

This section deals with those sensors which do not currently form the sensor fusion framework for ERTMS or CBTC train positioning system.

\subsubsection{Eddy current sensor}

Acharya et al. [18] suggested eddy current sensors as an auxiliary system for speed sensing device on railways. Lion Precision [19] identified that eddy current sensor is appropriate where there is a presence of liquid and a risk of contamination. In this context, Geistler and Bohringer [3] mentioned that eddy current sensors are more robust than tachometers in terms of weather and environmental conditions. In addition to non-destructive testing Geistler [20] pointed out that eddy current sensors are also be able to detect inhomogeneities in magnetic resistance along the track. Magnetic resistance is an important element to consider when designing track circuits. As magnetic resistance affects the current flow, it can jeopardise the detection of a train presence within a given track circuit.

\subsubsection{Global Positioning System (GPS)}

GPS is a satellite-based radio navigation system developed by the United States Department of Defense to provide accurate absolute positioning information over extended periods of time worldwide under all weather conditions [21]. GPS is currently used by the UK rolling stock maintenance [22] to identify the number of trips that a train is doing on a daily basis. This data then feeds into their regular maintenance regime which then builds up its maintenance schedule for all available rolling stock. Bajaj et al. [23] mentioned that GPS is rapidly becoming a universal utility as the cost of integrating the technology into vehicles, machinery, computers, and cellular phones is decreasing. Yao et al. [24] mentioned that the GPS solution is relatively stable and can maintain a consistent level of navigation functionality for a long period, but that it also suffers from multipath effects and its signal may easily be blocked or lost under certain environment.

Bhatt et al. [21] mentioned that although GPS has been widely used in land vehicle navigation systems, standalone GPS are unable to provide continuous and reliable navigation solutions in the presence of signal fading and/or blockage such as in urban areas. Hu et al. [25] studied real time adaptive algorithm on GPS satellite data processing for vehicle navigation where they mentioned that in practice, the number of measurements may vary from epoch to epoch timing frequency in a sensor fusion environment due to GPS signal being blocked off by obstacles or simply due to satellite unavailability. While discussing about the errors in GPS signal, Bajaj et al. [23] mentioned that a major source of error arises from the fact that radio signal speed is constant only in a vacuum. Errors due to multipath fading, which occurs when a signal bounces off a building or terrain before reaching the receiver's antenna, also can reduce the accuracy [23].

Differential GPS (DGPS) employs both roving receivers that make satellite position measurements and stationary receivers that use their position to compute signal timing [23]. While comparing GPS, DGPS and Assisted GPS (AGPS), Bajaj et al. [23] mentioned that AGPS provides a better accuracy typically less than $15 \mathrm{~m}$ and DGPS as less than $0.9 \mathrm{~m}$.

\subsubsection{Other Global Navigation Satellite System (GNSS) sensors}

GPS discussed in previous section is one form of GNSS. The other forms of GNSS are:

- $\quad$ GLONASS (Russia)

- Galileo (EU)

- Compass (China) 
Moreno et al. [26] mentioned that there are two major railway projects which are trying to validate satellite technology for railway safety services. The first one is project 3InSAT, led by Ansaldo STS and the other one is led by European Space Agency. They are now being tested in Sardinia with an intention to remove track circuits and GSM-R infrastructure using satellites for both location and communication.

While discussing about precise and reliable train positioning system, Albrecht et al. [27] stated that a modular on-board positioning system based on GNSS (Global Navigation Satellite System) is being developed in the German national research project PiLoNav. Jiang et al. [31] suggested that the competition between various means of transportation pushed the railways to look at the GNSS positioning as a more cost effective solution as compared to the use of trackside equipment. STARS project [4] also put forward a framework of train positioning system using GNSS.

The Indian Regional Navigation Satellite System (IRNSS) is an independent navigation system to be a constellation of seven satellites. Its primary service area will cover the Indian sub-continent extended by about $1500 \mathrm{~km}$. While carrying out a study on the effects of various environment factors on this system, Manjula and Raju [28] mentioned that under harsh conditions such as ionosphere scintillation and multipath effects, the performance of GNSS deteriorates leading to loss of phase lock and signal outages.

\section{FUSION FRAMEWORK}

From the above literature review, it is clear that a single sensor or a type of sensor is not good enough to carry out a train position estimate. Combination of sensors is the only way forward where the various noise profiles and sensor errors can be accommodated and at the same time provide an optimum train position estimate.

Kalman filter algorithm was chosen for fusing data from various sensors in order to get an acceptable state estimate. It has observation noise covariance $R$, state error covariance $Q$ and estimate error covariance $P$.

The simulation run that has been programmed in MATLAB is:

Run 1: The train starts from stationary to reach a speed of $350 \mathrm{~km} / \mathrm{hr}$ at an acceleration of $1.2 \mathrm{~m} / \mathrm{s}^{2}$.

Run 2: The train then coasts with acceleration $0 \mathrm{~m} / \mathrm{s}^{2}$ and reaches a distance of $6.5 \mathrm{~km}$.

Run 3: The train then brakes at a deceleration rate of $1.1 \mathrm{~m} / \mathrm{s}^{2}$ till it comes to a halt, hence speed reaches $0 \mathrm{~m} / \mathrm{s}$.

Standard deviation for the sensor noise as chosen from the manufacturer's catalogue are shown in Table 1.

Table 1: Sensor error standard deviation table.

\begin{tabular}{|l|l|}
\hline GPS & $0.2 \mathrm{~m}$ \\
\hline Doppler radar & $\begin{array}{l}0.027 \mathrm{~m} / \mathrm{s} \text { for speed }<\text { than } 100 \mathrm{~km} / \mathrm{hr} \\
0.01 \% \text { of true speed for speed }>\text { than } 100 \mathrm{~km} / \mathrm{hr}\end{array}$ \\
\hline Tachometer & $1 \%$ of true speed \\
\hline Accelerometer & $3 \%$ of true acceleration \\
\hline Balise & $0: 25 \mathrm{~m}$ \\
\hline
\end{tabular}


Nine sensor frameworks are chosen as shown in Table 2.

Table 2: Sensor fusion framework.

\begin{tabular}{|l|c|c|c|c|c|}
\hline Fusion framework & GPS & Doppler & Accelerometer & Tacho & Balise \\
\hline Frm 1 & $\sqrt{ }$ & $\sqrt{ }$ & & & \\
\hline Frm 2 & $\sqrt{ }$ & & $\sqrt{ }$ & & \\
\hline Frm 3 & & $\sqrt{ }$ & & $\sqrt{ }$ & $\sqrt{ }$ \\
\hline Frm 4 & & & & $\sqrt{ }$ & $\sqrt{ }$ \\
\hline Frm 5 & & $\sqrt{ }$ & & & $\sqrt{ }$ \\
\hline Frm 6 & $\sqrt{ }$ & & & $\sqrt{ }$ & \\
\hline Frm 7 & & $\sqrt{ }$ & & $\sqrt{ }$ & \\
\hline Frm 8 & $\sqrt{ }$ & $\sqrt{ }$ & & $\sqrt{ }$ & \\
\hline Frm 9 & $\sqrt{ }$ & $\sqrt{ }$ & $\sqrt{ }$ & $\sqrt{ }$ & \\
\hline
\end{tabular}

The rationale for considering the sensors as shown in Table 2 is driven by the literature review carried out in Section 2.

Drawing the rationale for tachometer being the robust and reliable sensor [11] used on railways and currently been within the sensor fusion framework, we have considered the tachometer to be within the framework for most experiments. The same rationale applies to the doppler sensor also.

Accelerometer on the other hand is currently only used on some CBTC systems and given the bias errors [6] and the issue of measurement frequency [8], we do not consider it as our preferred sensor.

GPS on the other hand, although do not form the part of the existing rail sensor fusion framework, it is used for rolling stock maintenance as identified in Section 2.5.2. It provides relatively stable data [24] and is currently extensively used in road vehicle navigation.

Balise being an external sensor do not form part of the sensor fusion framework which sits within the train. Therefore, they provide location information periodically as set by the designer and not by the system. As identified, there is a high chance that the train at a high speed might miss the balise which doesn't help in estimating the train location which is the primary aim of this research. Hence use of balise in this framework is kept minimal.

Using the above sensor framework, we ran traditional Kalman filter assuming all the a priori assumptions and filter properties are correct using MATLAB software for each of the sensor combination.

\section{ANALYSIS}

\subsection{Analysis of filter response in terms of variance covariance}

The filter error variance covariance $P$ is the measure of the filter stability. As pointed out by Groves [29], it records the correlation between the state estimates and the uncertainties. In a way it provides the best knowledge of the system dynamics at any instant $t$. As the $P$ approaches zero, the filter puts less emphasis on the measurement [30].

The diagonal matrices of $P$ are the variance covariances of "location", "speed" and "acceleration" respectively. Analysing the results of P11, P22 and P33 we find the following: 
Doppler-Tachometer combination is not the best of the sensor framework for any of the state estimates. While it is worse for location state estimate, it is not providing the best estimate for speed and acceleration either.

GPS-Tachometer-Doppler-Accelerometer produces the best estimate for position, but with an increasing speed, the variance covariance matrix is increasing which indicates that with faster train speed and high acceleration, the filter might tend to diverge. A similar property is shown for the speed estimate also. However, for the acceleration estimate, the sensor framework exhibits better result.

The GPS-Doppler combination shows a similar performance for the location estimate to that of GPS-Tachometer-Doppler-Accelerometer combination, however it doesn't show any sign of dipping during the "zero" acceleration phase. This may due to the fact that it is not getting acceleration observation data and hence is solely relying on the filter. The sensor framework however is not exhibiting the best of the results for the speed and acceleration state estimate.

GPS-Accelerometer fusion framework exhibits the most stable filter for the location estimate. It doesn't show any change due to growing speed. The filter also shows stable behaviour for the speed and acceleration state estimates. It is the best sensor combination for the acceleration state estimate.

Doppler-Tachometer-balise is the current sensor fusion framework which is used ERTMS and in most CBTC systems. It exhibits a stable position estimates only once it receives the balise reading. However, it doesn't exhibit a good performance for speed estimate and shows a tendency to a growing variance covariance. This suggests it might not be the best sensor fusion framework for high speed rail network and high performing, high accelerating trains. Therefore, for mass transit network, where the trains need to accelerate and brake fast, this sensor framework may not be the best combination. The acceleration estimate is not impressive either.

The Tachometer-balise sensor framework exhibits a high degree of dependency on the balise reading, particularly for the location estimate. For the speed and acceleration estimate, this is the worst sensor combination as evident from the three graphs.

The Doppler-balise combination exhibits similar dependency on the balise reading as that of Tachometer-balise framework. But it is a better fusion combination than the Tachometer-balise framework. The speed estimate is not what expected given it has got a speed sensor. The speed estimate exhibits a trend towards growing uncertainty as the speed increases. While it is not the worst sensor combination for the acceleration state estimate, it is not the best either.

GPS-Tachometer combination does not exhibit the best estimate in any of the state estimates. While it is not the worst, it is not good either.

GPS-Tachometer-Doppler combination is very similar to GPS-Doppler in terms of position state estimate. However, it is not a good sensor combination for speed and acceleration state estimate.

\subsection{Analysis in terms of error recording}

Analysing the filter from the error perspective, we choose the best filter which gives least error.

Out of the nine sensor combinations as identified in Table 2, three sensor combinations give the best estimate in terms of least location estimate error. They are GPS-Doppler, GPS-Tachometer-Doppler and GPS-Tachometer-Doppler-Accelerometer. 
At the beginning of the filter run, the GPS-Doppler is the most stable framework. But all the three sensor frameworks stabilise within ten seconds of the filter run and produces almost same location estimate error.

The change of acceleration occurs at 81 second and 107 second. Fig. 1 suggests that GPS-Doppler and GPS-Tachometer-Doppler sensor combination provides better estimate than the GPS-Tachometer-Doppler-Accelerometer sensor combination.

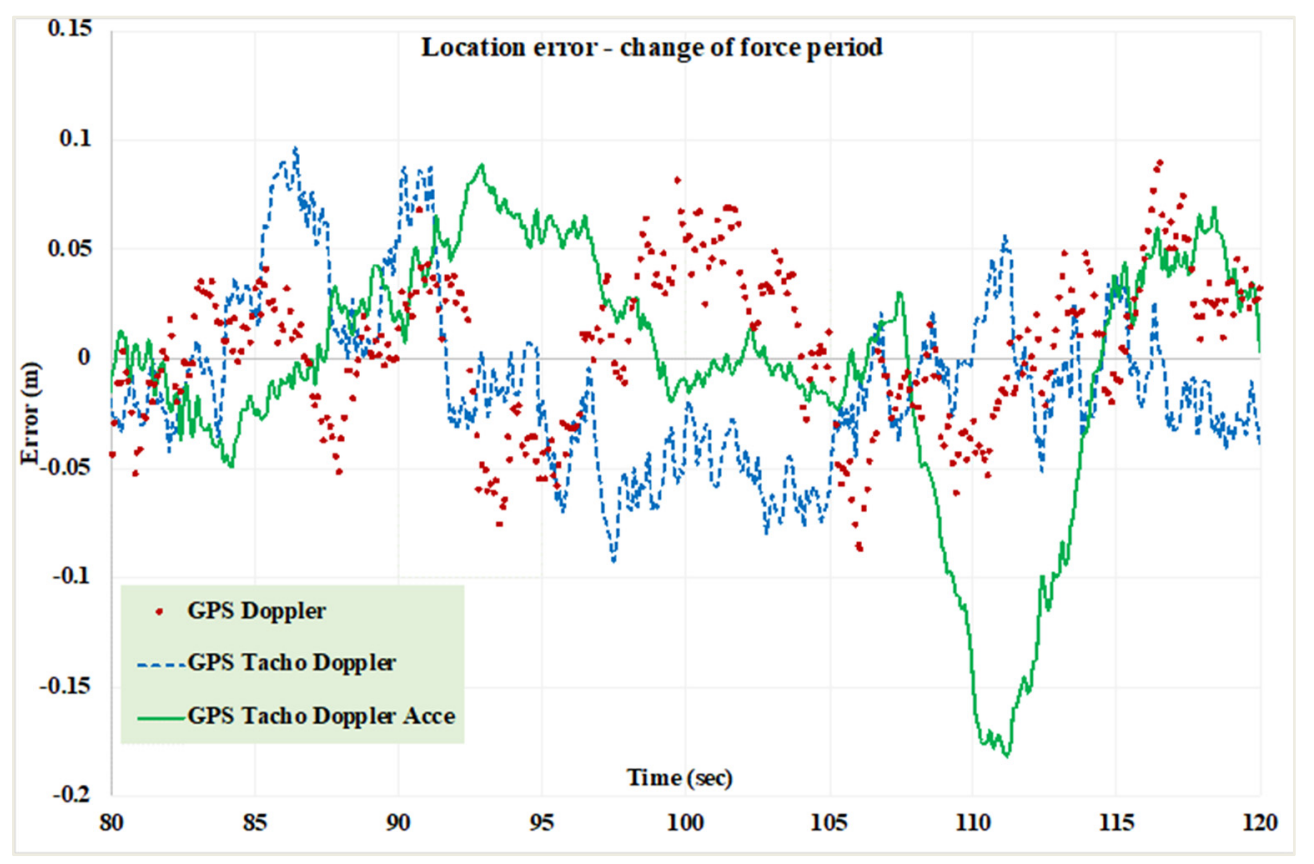

Figure 1: Comparison of location error during force change.

At the end of the mission, when the filter parameters are quite established and the train is coming to a halt, the three sensors are performing better than any other sensor combinations.

Similar comparison was carried out for speed and acceleration. For the speed estimate (Fig. 2), it seems that the best sensor fusion frameworks are GPS-Accelerometer, Doppler-Tachometer and GPS-Doppler-Tachometer-Accelerometer. During the change of acceleration phase, the best estimate is shown by GPS-Accelerometer and also by Doppler-Tachometer.

Fig. 2 and Fig. 3 show the speed state estimate error and acceleration state estimate error as recorded for the best combination of sensors. The best sensor framework that seems to produce the best acceleration estimate (Fig. 3) are GPS-Accelerometer and GPS-Tachometer-Doppler-Accelerometer sensor framework. While the GPS-Accelerometer framework produces better estimate during the initial phase of the filter run, they produce almost similar estimate during the final phase of the filter when the system parameters are well established. During the acceleration change phase, while both the framework produces similar error estimate, both exhibits zero error during zero acceleration phase. This suggests that including a sensor which can measure acceleration within the sensor fusion framework 
provides better acceleration estimate. Both exhibits sudden change of error at the point when there is a change of acceleration from 0 to $-1.1 \mathrm{~m} / \mathrm{s}^{2}$.

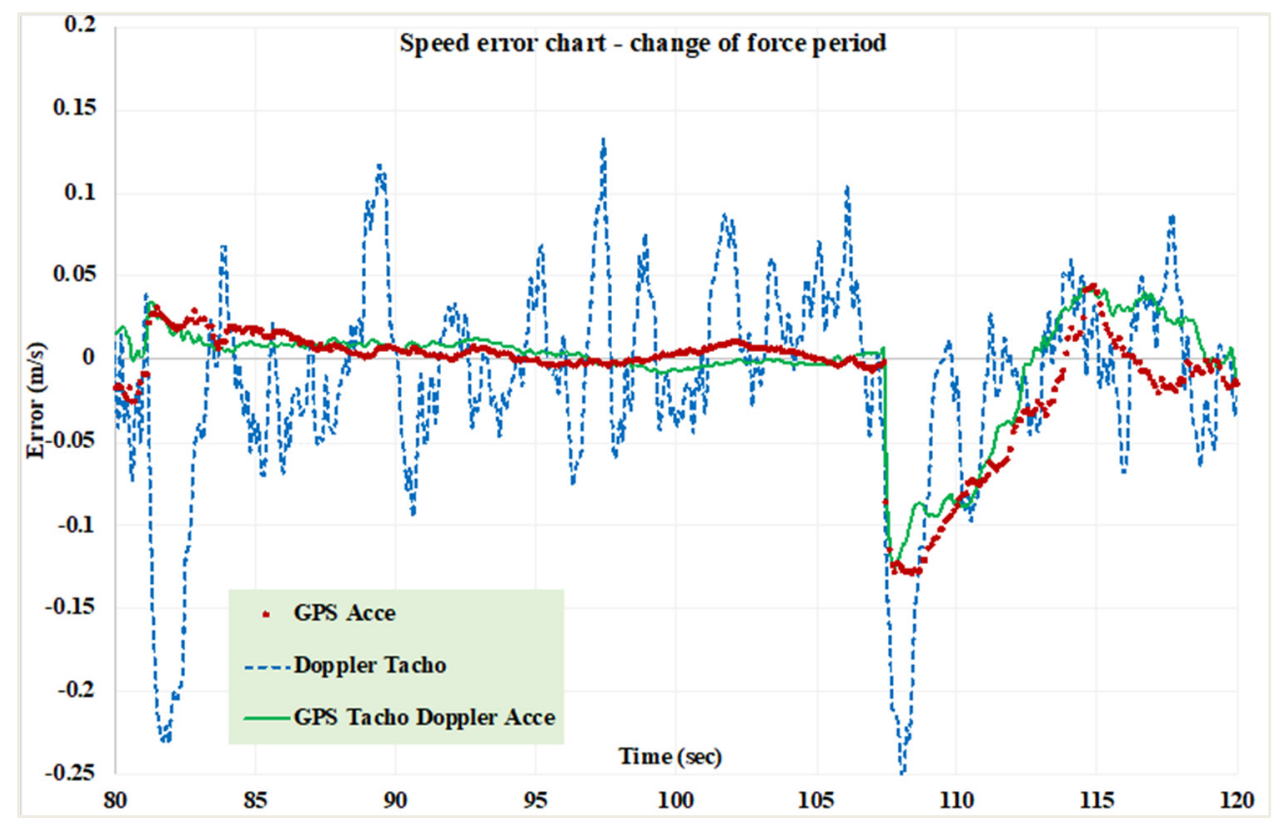

Figure 2: Comparison of speed error during force change.

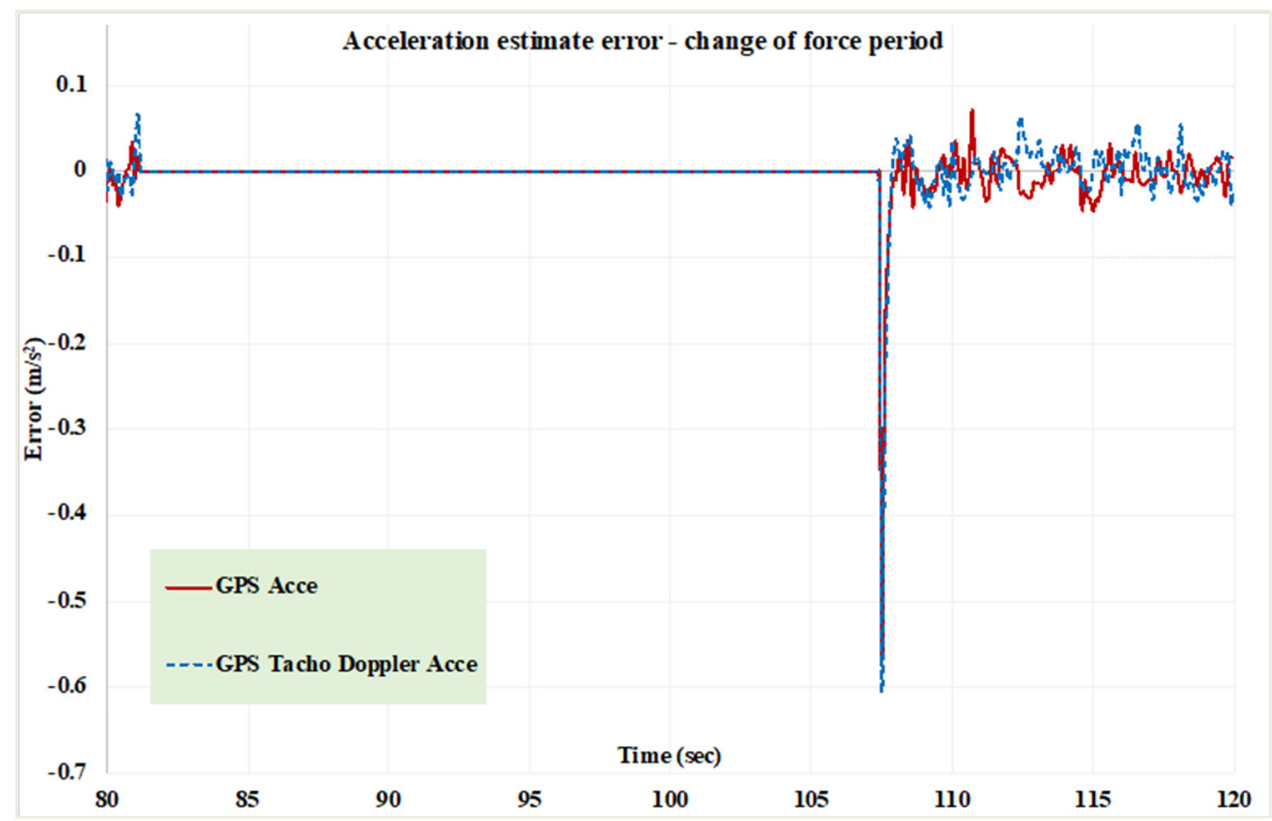

Figure 3: Comparison of acceleration during force change. 


\section{CONCLUSION}

Judging the behaviour of the filter with various sensor combinations, we can draw the following conclusion: GPS is the chosen location sensor than that of balise. The rationale is wherever GPS has been fused with other sensor, the filter provides a better position estimate. Fusing an accelerometer with GPS always provides a better state estimate for all location, speed and acceleration.

Use of tachometers in a sensor framework effects the overall filter accuracy including that of speed estimate. None of the sensor fusion framework with tachometer provides a better speed estimate unless it is fused with GPS and accelerometer. While the traditional Doppler-Tachometer-balise combination provides a fairly good location estimate, it is not good as the estimate that we get by fusing accelerometer or GPS with it. Interestingly the combination of Doppler-balise and Doppler-Tachometer-balise provides the same state estimate for all location, speed and acceleration. This proves that the tachometer has no impact on the estimate even on speed. It is not adding any value to the filter estimate.

The accelerometer sensor which is currently scarcely used due to the reason explained in Section 2.3 turned out to provide fairly good state estimate. Therefore, further experiments will be carried out including accelerometer sensors.

None of the experiments involving balise provides any better state estimate. The indication is where balise is solely used for the purpose of location observation along with Doppler and Tachometer, the filter tends to increase its uncertainties till it gets a reading from the balise. Given the fact that the balise are mounted on the track at a fairly specific interval, the system is only constrained to receive balise information every specific distance of travel. This suggests that any incident of missing balise will lead to growing uncertainties within the location state estimate.

Therefore, the conclusions are:

1. GPS is the chosen sensor for location observation;

2. Doppler is the chosen sensor for speed observation;

3. Accelerometer is the chosen sensor for acceleration observation;

4. More simulations incorporating failure modelling within the sensor fusion framework; and

5. Standardise sensors and the fusion architecture for any ERTMS solution in order to achieve interoperability.

\section{REFERENCES}

[1] Mirabadi, A., Mort, N. \& Schmid, F., Application of sensor fusion to railway systems. International Conference on Multisensor Fusion and Integration for Intelligent Systems, pp. 185-192, 1996.

[2] Malvezzi, M. et al., Train position and speed estimation by integration of odometers and IMUs. 9th World Congress on Railway Research, pp. 22-26, 2011.

[3] Geistler, A. \& Bohringer, F., Robust velocity measurement for railway applications by fusing eddy current sensor signals. IEEE Intelligent Vehicles Symposium Proceedings, pp. 664-669, 2004.

[4] Bazant, L., Toma, A. \& Mocek, H., Description of Methodology for Data Record Sorting and Saving Satellite Technology for Advanced Railway Signalling. Tech. report 687414, p. 114, 2017.

[5] Transit Cooperative Research Programme (TCRP), Tech. report, August. pp. 17-64, 1989. 
[6] Mirabadi, A., Mort, N. \& Schmid, F., Design of fault tolerant train navigation systems. Proceedings of the American Control Conference, San Diego, California, June, pp. 104-108, 1999.

[7] Heide, P., Magori, V. \& Schwarte, R., Coded 24 GHz Doppler radar sensors: A new approach to high-precision vehicle position and groundspeed sensing in railway and automobile applications. IEEE NTC 1995 - Microwave Systems Conference, Conference Proceedings, pp. 101-104, 1995.

[8] Malvezzi, M., Allotta, B. \& Rinchi, M., Odometric estimation for automatic train protection and control systems. Vehicle System Dynamics 49.5, pp. 723-739, 2011.

[9] Wrobel, S. Multifunction LIDAR sensors for non contact speed and track geometry measurement in rail vehicles. PhD thesis, pp. 1689-1699, 2013.

[10] Maybeck, P.S., Stochastic models, estimation, and control. New York, vol. 1, pp. 116, 1979. http://www.cs.unc.edu/ welch/media/pdf/.

[11] Malvezzi, M. et al., Train position and speed estimation by integration of odometers and IMUs. 9th World Congress on Railway Research, May, pp. 22-26, 2011.

[12] Vettori, G. et al., Innovative management of wheel-rail adhesion conditions in localization algorithms for the automatic train protection. Chemical Engineering Transactions, 33.2, p. 685, 2013.

[13] Shieh, J., Huber, J.A., Fleck, N.A. \& Ashby, M.F., The selection of sensors. Progress in Materials Science, 46(3-4), pp. 461-504, 2001.

[14] Endevco, Steps to selecting the right accelerometer.

[15] Sharma, R. \& Lourde, R.M., Crosstalk reduction in balise and infill loops in automatic train control. INES 2007 - 11th International Conference on Intelligent Engineering Systems Proceedings, pp. 39-44, 2007.

[16] Mendizabal, J. et al., Communication Technologies for Vehicles, vol. 7865, pp. 27-39, 2013.

[17] UNISIG, Principles. System Requirements Specification, Chapter 3, 2012.

[18] Acharya, A., Sadhu, S. \& Ghoshal, T.K., Train localization and parting detection using data fusion. Transportation Research Part C: Emerging Technologies, 19(1), pp. 7584, 2011.

[19] Lion Precision, Comparing non - contact sensors: Capacitive vs. eddy - current sensors, 2013.

[20] Geistler, A., Train location with eddy current sensors. CompRail 2002, pp. 12-14, 2002.

[21] Bhatt, D. et al., Expert systems with applications. A novel hybrid fusion algorithm to bridge the period of GPS outages using low-cost INS. Elsevier/Expert Systems With Applications, 41(5), pp. 2166-2173, 2014.

[22] Bertran, E. \& Delgado-Penín, J.A., On the use of GPS receivers in railway environments. IEEE Transactions on Vehicular Technology, 53(5), pp. 1452-1460, 2004.

[23] Bajaj, R., Ranaweera, S.L. \& Agrawal, D.P., GPS: Location tracking technology. Computing in Science \& Engineering, pp. 92-94, 2002.

[24] Yao, Y. et al., A hybrid fusion algorithm for GPS/ NS integration during GPS outages. Elsevier/Measurement, 103, pp. 42-51, 2017.

[25] Hu, Y., Duan, Z. \& Zhou, D., Estimation fusion with general asynchronous multi-rate sensors. IEEE Transactions on Aerospace and Electronic Systems, 46(4), pp. 2090$2102,2010$. 
64 Computers in Railways XVII

[26] Moreno, J. et al., A survey on future railway radio communications services: Challenges and opportunities. IEEE Communications Magazine, 53(10), pp. 62-68, 2015.

[27] Albrecht, T., Lüddecke, K. \& Zimmermann, J., A precise and reliable train positioning system and its use for automation of train operation. IEEE ICIRT 2013 - Proceedings: IEEE International Conference on Intelligent Rail Transportation, pp. 134-139, 2013.

[28] Manjula, T.R. \& Raju, G., A comprehensive study of linear Kalman filter based tracking techniques under ionosphere scintillation. Procedia Technology, 25, pp. 427434, 2016.

[29] Groves, P.D., Principles of GNSS, Inertial, and Multisensor Integrated, Technology, 2008.

[30] Welch, G. \& Bishop, G., An introduction to the Kalman filter. SIGGRAPH, pp. 1-81, 2001.

[31] Jiang, L., Bai Gen, C. \& Tao Jian, W., A CKF based GNSS/INS train integrated positioning method. 2010 IEEE International Conference on Mechatronics and Automation, ICMA 2010, IEEE, pp. 1686-1689, 2010. 